\title{
Mit einer Stimme sprechen: Erfolgsmodell Ärztering?
}

Im niederrheinischen Rees haben sich 17 Ärzte im Ärztering Rees zusammengeschlossen, um die medizinische Versorgung trotz hoher Altersstruktur und Nachwuchsmangels zu sichern. Erste Erfolge gibt es bereits.

- Zusammenarbeit ist alles, auch und gerade in strukturschwachen Regionen. Elf Praxen mit 17 Ärzten im niederrheinischen Rees versuchen die Kooperation über einen Ärztering statt über ein Ärztenetz. Es zeigen sich bereits Erfolge, so der Sprecher des Netzwerks, Dr. Michal ElNounou: „Das beste Beispiel ist, dass ein Kollege seine Patienten für das LangzeitEKG früher immer in eine Praxis ins 20 Kilometer entfernte Wesel geschickt hat, weil er nicht wusste, dass ich das anbiete."

Die beteiligten Ärzte setzen sich alle vier bis sechs Wochen zusammen. Ein Vorteil der Kooperation ist, dass die Me- diziner gegenüber den Krankenkassen und Kliniken mit einer Stimme auftreten.

\section{Nicht mal mehr \\ Thorax-Röntgen}

Aktuell sucht der Ärztering nach einer Möglichkeit, wie wieder Röntgenuntersuchungen der Lunge angeboten werden können. Das ist derzeit nicht möglich, weil die geriatrische Abteilung der Klinik weggezogen ist. „Das zeigt, wie sich die Situation auf dem Land entwickelt", sagt El-Nounou.

El-Nounou hofft, dass der Ärztering dazu beitragen kann, Rees für Nachwuchsmediziner attraktiver zu machen. Noch gebe es genügend Ärzte vor Ort, aber das Problem werde bald akuter, erklärt El-Nounou. Viele Ärzte in Rees seien bereits im Rentenalter oder kurz davor. „Drei der vier Hausärzte in den Reeser Vororten sind über 60 Jahre alt", sagt der 35-Jährige.

Für die Zukunft plant der Ärztering eine noch engere Abstimmung unter seinen Mitgliedern. „Wir wollen Behandlungspfade von der Voruntersuchung bis zur Behandlung beim Facharzt festlegen“, sagt El-Nounou. Geht das Konzept auf, könnten Behandlungen besser strukturiert werden und sich die Wartezeiten für Patienten verkürzen.

TAU =

\section{Ärzte aus Spanien}

\section{Herzlich willkommen in Sachsen!}

\section{Sie suchen eine neue Chance in Deutschland: Ärzte aus Spanien über- nehmen Praxen in Sachsen, für die sich trotz langer Suche kein Nachfol- ger gefunden hat. Die KV freut sich.}

- Wie die KV Sachsen jetzt mitteilte, wurden erstmals Ärzte von der iberischen Halbinsel nach Sachsen gelotst, um Praxen zu übernehmen, für die sich bisher keine Nachfolger fanden.

Bei der Ansiedlung handle es sich um ein Modellprojekt, so die Kammer, weitere Abwerbungen können folgen. „Es wurden zunächst acht Praxen ausgewählt, deren Inhaber seit Langem einen Praxisnachfolger suchen und eine große Anzahl von Patienten versorgen“", so die KV. Die ersten drei Ärzte unterschrieben bereits, ,weitere Verträge stehen kurz vor dem Abschluss", versichert die KV.

\section{Wirtschaftliche Gründe}

Die Ärzte aus dem krisengebeutelten Spanien entscheiden sich vor allem aus wirtschaftlichen Gründen für eine Übersiedlung. Die Kosten für die Vermittlung müssen sie später selbst aufbringen. Es handle sich, so die KV, um Kollegen in ungekündigter Stellung. Sie verpflichten sich, mindestens vier Jahre am neuen Standort in Sachsen niedergelassen zu bleiben.

Anfang August begannen drei spanische Ärzte einen Sprachkurs, nach einem einwöchigen Praktikum beim Praxisinhaber werden sie ihre Arbeit aufnehmen. „Die meisten Kandidaten werden bereits jetzt in den Orten wohnen, wo sie später arbeiten. Die Integration der Familien und der frühe und kontinuierliche Kontakt zu den potenziellen Patienten stehen dabei im Mittelpunkt", so die KV Sachsen (s. dazu auch S. 9). 